\title{
A Circuit Breaker Reliability Model for Restoration Planning Considering Risk of Communication Outage
}

\author{
F. Edström, L. Söder, Royal Institute of Technology, Stockholm, Sweden
}

\begin{abstract}
A large scale blackout is a long term loss of electricity over a large geographical area. In order to succeed with the restoration, the electric utility SCADA system must be highly reliable not only in normal operation but also during a black out. The main contribution of this paper is a circuit breaker (CB) reliability model to be used in restoration planning. The model consider both the reliability of a SCADA system and the reliability of a communication system during a power outage. If a remote control of $\mathrm{CB}$ fails, due to battery undersizing, it is assumed in the paper that a manual operation of $\mathrm{CB}$ can be performed if field personal can communicate with the TSO head-quarter. The evaluation of the reliability to operate $\mathrm{CB}$ is performed by solving the so called s-t reliability problem for a graph with failure prone components. The method is applied in a case study where the SCADA architecture for an IEEE reliability test system is modeled.
\end{abstract}

Index Terms-SCADA, black out, Restoration, GSM network,

\section{INTRODUCTION}

B ETWEEN the fourth and tenth of January 1998, eastern Canada was hit by the biggest ice storm in modern Canadian history [1]. Due to heavy ice formation on the transmission lines there were severe damage on the grid which lead to a large black out affecting 4 million people.

The restoration process following a large black out is a complex and time consuming task involving many actors, and the need for efficient restoration strategies is obvious. The time it takes to restore a system is critical, since important systems runs on backup power during the power outage. For example, a remote control of circuit breakers depends on local backup power at substations. If the power outage at a substation is prolonged the backup power might be insufficient and the circuit breakers must be operated manually. Since a manual operation of a circuit breaker is more time consuming than a remote control, the restoration will be delayed. Moreover, a manual operation of circuit breakers, or a restoration of broken transmission lines, depends on communication between the transmission system operator and field personal. During a blackout communication depends on backup power as well.

Power system restoration is an area extensively covered in the literature, see for example [2] by Adibi et al. which provides an excellent overview of the field. However, [2] shows that little research has been focused on the impact of limited backup power during extended blackouts. To extend the research in this area, the authors have in [3] developed a method to analyze the impact of remote control failure due to battery undersizing at substations on power system restoration time. However, no consideration is taken to the reliability in the SCADA system in this work. A method to analyze the reliability of the electric utility SCADA system has been presented by A.G. Bruce in [4]. The paper focuses on the SCADA systems contribution to load curtailment in the power system, but the work does not consider limited backup power during a power outage. Problems with backup power during the restoration at substations and in the communication systems are well known and, for example, discussed in [5], [6] and [7]. Restoration planning has been discussed by Bouchey et al. in [8] and by Adibi et al. in [9]. To understand the reliability to operate $\mathrm{CB}$ during a power outage, new models including backup power both at substations and in the communication system are needed. These models can be used to determine how fast a restoration must proceed to avoid issues associated with loss of backup power.

This paper develops a CB reliability model to be used in restoration planning. The model includes both the reliability to operate CB through the SCADA system and through a manual operation by field personnel. In case of a remote control failure through the SCADA system, it is assumed that field personnel can operate the $\mathrm{CB}$ manually if there is a system for remote voice communication between the TSO and the field personnel. The SCADA architecture presented in [4] where master station, SCADA data communications network, remote terminal units and the wide area network (WAN) is present, is applied to the model in a numerical example. Furthermore, it is also assumed that there is a total black out and that the substations and the communication network for remote communication with field personnel runs on limited backup power. As long as the communication network works, it offers a way to communicate with field personnel and therefore a possibility for a manual operation of $\mathrm{CB}$.

This paper is structured into seven sections. Section two gives an introduction to backup power. Section three describes a remote communication system and how it depends on backup power during a black out. A simple reliability model for the communication system network is derived in this section. Section four describes the reliability of a SCADA network. Section five derives the reliability to operate CB. Section six shows a numerical example and section seven gives conclusions.

\section{BACKUP POWER}

During power outage or in case of a failure, backup power is needed at substations and in the telecommunication system to 
support the basic functions. At substations, backup power are for example needed for climate control, lighting, cooling systems and communication equipment. In a telecommunication system backup power are needed to maintain communication. These loads are supported by the power system during normal operation. In case of power outage, common backup power used in telecommunication systems and in power systems, are batteries and diesel generators. Due to the great number of substations, both on transmission level and on distribution level in a large power system, and the number of base stations in a large telecommunication system, it is not economically feasible to invest in diesel generators as backup power everywhere. The consequence of this is that a efficient restoration of a power system is heavily depended in batteries as backup power. One of the most common type of battery used for backup power purposes is the lead-acid battery, because it is a cheap alternative and has a long life, see [10], [11] and [12]. Another common battery is the Nickel-Cadmium battery. To understand the reliability to operate $\mathrm{CB}$ during a power outage, it is therefore necessary to understand the impact of batteries as backup power in both the telecommunication system and in the power system.

\section{A. Substation load profile}

The capacity for a battery and the load determines the time a system can be powered. A typical loading profile for a substation during a power outage can be divided into three time periods.

- Time period I: The backup over current relay operates, which in turn trips a high side circuit switcher and low side breaker. Normally this time period is assumed to be one or two minutes.

- Time period II: During the power outage batteries has to give power to indicating lamps, annunciators and emergency lights, cooling and heating system and so on.

- Time period III: The main circuit breaker at the substation is closed. This time is similar to time period one and is assumed to be one or two minutes.

It is important to note that there is a large current draw at the end of the duty cycle which could lead to capacity problem and a failure when trying to operate the CB. Without the battery capacity at this time the battery charger has to be re-powered or a manual operation is needed. To avoid these issues sizing of batteries as backup power purpose is therefore an important task. ANSI/IEEE Standard 485, IEEE Recommended Practice for Sizing Large Lead Storage Batteries for Generating Stations and Substations, [13], gives a guide to sizing the batteries. However, extended power outage might still occur and lead to capacity problems.

\section{B. Battery capacity}

There are several factors that affects the battery capacity and the battery life time [10]; battery temperature, frequency of use, minimum operating voltage, future load growth, design margin and life margin. For example, a lead-acid battery should be maintained at $25 \mathrm{C}$ under ideal conditions. This is maintained through air conditioning and heating. At lower temperatures the battery capacity is reduced significantly. During an extended outage the temperature might drop, or increase, depending on the ambient temperature and therefore affect the battery capacity. Furthermore, when a battery is installed the capacity might be as low as 90 percent of its rated capacity. The capacity will then increase during the first years, followed by a drop to 80 percent of its rated capacity at the end of the its life. Considering this example, it is not unreasonable to assume a stochastic capacity for the battery. If a large quantity of batteries and an large number of loading profiles are considered, it is not unreasonable to assume a normal distribution for the time a battery can support a load due to the central limit theorem.

\section{Battery maintenance}

Maintenance of the batteries and the battery charger are essential. It should include inspection of the batteries and charger, testing for the specific gravity of the electrolyte and load testing. However, failure might still occur. An illustrative example is given in [10] where a battery charger failure resulted in permanent damage to the battery at a substation. This failure resulted in a loss of a 138-24.9 kV transformer when a bird caused a flash over on a $25-\mathrm{kV}$ bus. The protective relays sensed the fault and attempted to trip a $138 \mathrm{kV}$ circuit switcher. But the battery had been damage due to a deep discharge when the battery charger failed. It did not have the capacity to trip the circuit breaker and this resulted in total loss of a $10 \mathrm{MVA}, 138-24.9 \mathrm{kV}$ transformer and a major damage to the $25-\mathrm{kV}$ bus. This happed even though the battery and charger were inspected weekly.

Considering the example, it is not unreasonable to assume that there is a probability that the battery will fail supporting the load.

\section{Formalization}

To formalize the batteries ability to support the load, let

$$
\Phi=\left\{\begin{array}{cc}
1 & \text { battery can support the load } \\
0 & \text { can not }
\end{array}\right.
$$

Further, let $B_{i}$ be the capacity for battery $i, p_{b}$ be the probability that the battery and the equipment needed for power supply will work, and let $L(t)$ denote the load. The probability for power supply from battery $i$ at time $t$ after a black out is given by

$$
P(\Phi=1)=p_{b} P\left(B_{i}>\int_{0}^{t} L(t) d t\right)
$$

Note that $B \sim \mathcal{N}\left(\mu, \sigma^{2}\right)$.

\section{MAnUAL OPERATION OF CB}

During a black out, the communication between a transmission system operator and field personnel depends on a remote communication system like the global system for mobile communications (GSM) network. Remote communication could 
also be maintained through satellite based communication systems or radio based systems. Since the GSM system is used world wide, it is assumed to be the preferred and only channel for remote communication in this study.

Communication between the TSO head-quarter and field personnel is important since it is essential to know which CB that has to be closed and when. Therefore, it is assumed that a manual operation can only be executed as long as there exists a communication channel between the TSO head-quarter and field personnel. A simple reliability model can be derived from the structure of the GSM network.

\section{A. The GSM Network}

The GSM system is well descried in the literature. See for example [14] and [15]. A principal structure of a GSM network is showed in figure 3 .

A GSM network consists in short of, base transceiver stations (BTS), base station controllers (BSC), base station subsystems (BSS), mobile switching centers (MSC), gateway mobile switching center (GMSC) and home location register (HLR). The BTS communicates wireless with the mobile phones. The BSC monitors and controls a number of BTS's. The BTS's and the BSC forms a BSS. At the highest level are the MSC. A number of BSS are monitored and controlled by a MSC. The MSC's are connected to other networks like the PSTN through a GMSC. The MSC and GMSC has access to databases that contain information about the mobile users like the home location register (HLR).

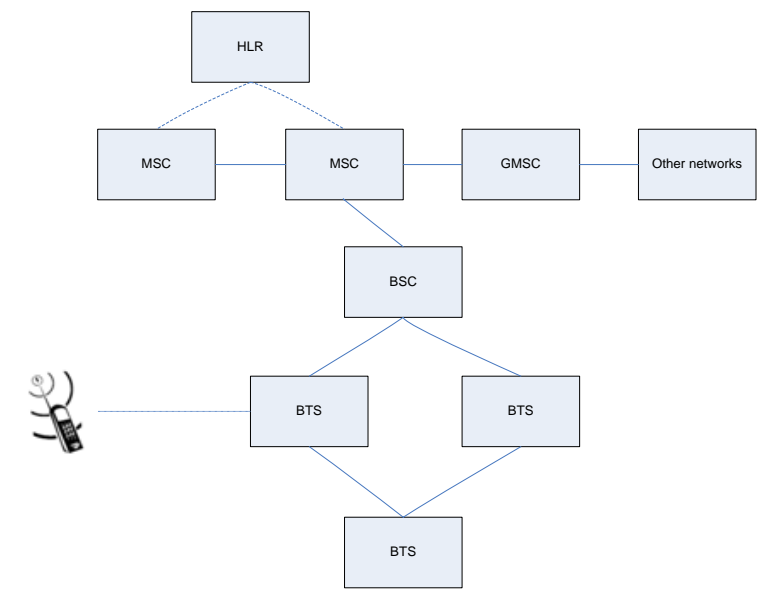

Fig. 1. Principal structure for a GSM network

How a call is routed from the public switched telephone network (PSTN) to a cell phone in the GSM network is described in detailed in [14]. A call is routed through the PSTN to the closest GMSC within the mobile's public land mobile network (PLMN). The GMSC interrogates the mobiles HLR to route the call to the MSC of the mobile at the time. The MSC pages all BSS of the location area, as the exact location is unknown. After response the current BSS is located and the call is set up.

To construct a simple reliability model for the existence of a remote communication channel to field personnel during a black out three assumptions are made;
1) A call from the TSO head-quarter has to be directed through the PSTN, a GMSC, a MSC, a BSC and a BTS.

2) A successful call from the TSO head-quarter to field personnel depends on whether the MSC, BSC and the BTS at the location has backup power or not.

3) The BTS's has the shortest backup power and runs on lead-acid batteries with the same properties as described above. This assumption is supported by [11], [12] and [16].

Taking these assumptions into account the probability for the existence of a remote communication channel between the TSO head-quarter and field personnel at a time, $t$, after a black out can be approximated with equation 2 , where $L(t)$ is the load at a BTS. A realization of equation 2 using, $\mu=2$, $\sigma^{2}=0.2, p_{b}=0.95$ and $L(t)=1$ is showed in figure 4 .

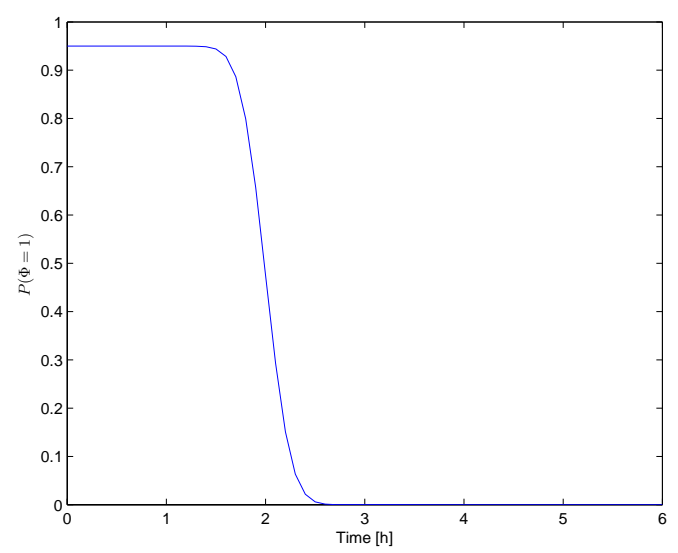

Fig. 2. Example for the existence of a communication channel

A manual operation of $\mathrm{CB}$ also depends on personnel with the right knowledge and experience of how to perform a manual operation. Furthermore, a person also has to travel to the actual substation. If the black out is caused by a heavy storm, the roads leading to the substation might be unavailable. Therefore, it is not unreasonable to assume that the probability that a manual operation will succeed is given by

$$
P(\Theta=1)=P(\Phi=1) p_{p} p_{r}
$$

where $p_{p}$ is availability of field personnel with the knowledge of how to operate $\mathrm{CB}$ manually and $p_{r}$ is the availability of roads leading to the substation and

$$
\Theta=\left\{\begin{array}{cc}
1 & \text { manual operation will succeed } \\
0 & \text { will not }
\end{array}\right.
$$

\section{REMOTE CONTROL OF CB}

A remote control of $\mathrm{CB}$ is made through a SCADA system. A SCADA systems contains several components which each has a availability, see figure 5 for an example of a SCADA network. Computing the reliability between two components in a network, in this case the workstation located at the TSO head-quarter and the $\mathrm{CB}$, is called the s-t reliability problem and is known to be a be P-complete. To deal with the 
computational complexity, two compromises are usually made. The first compromise is to deal with reliability bounds instead of exact solutions. The second compromise is to simplify the analysis with perfect reliable components.

To analyze the reliability to operate $\mathrm{CB}$ through the SCADA network, the algorithm presented in [17] is used in this paper. The algorithm finds the $m$ most probable state spaces for the system. Reliability bounds are formed using equation 5, 6, and 7. Since the algorithm presented in [17] finds the most probable state spaces it is possible to reduce the reliability bound and to increase the computational speed by introducing perfect reliable components when dealing with SCADA network. This is done by assuming that components which is used to control $C B_{j}$ but not included in the control of $C B_{i}$ is set to be perfect reliable when analyzing $C B_{i}$. Since a component can be in either working- or fault mode, the number of states the system have are $2^{l}$, where $l$ is the number of failure prone components. If $n$ components are included in the operation of $C B_{i}$, but not in the operation of $C B_{j}$, the computational complexity is hence reduced to $2^{l-n}$. Let $S_{k}$ denote the state space and let $P\left(S_{k}\right)$ denote the probability for the particular state space.

Further, let

$$
C_{i}\left(S_{k}\right)=\left\{\begin{array}{cc}
1 & \text { if it is possible to operate } C B_{i} \\
0 & \text { in state space } S_{k} \\
& \text { if not }
\end{array}\right.
$$

The reliability bounds is then found from

$$
\begin{aligned}
K_{L i} & =\sum_{k=1}^{m} P\left(S_{k}\right) C_{i}\left(S_{k}\right) \\
K_{U i} & =\sum_{k=1}^{m} P\left(S_{k}\right) C_{i}\left(S_{k}\right)+\left(1-\sum_{k=1}^{m} P\left(S_{k}\right)\right)
\end{aligned}
$$

where $K_{L i}$ is the lower boundary and $K_{U i}$ is the upper reliability boundary.

The algorithm to analyze the reliability to operate $\mathrm{CB}$ at substation $i$ at time $t$ is presented below.

1) Find the probability that the batteries can support the load at time $t$.

2) Set reliability to 1 for components which is not included in the control of bus $i$.

3) Find the $m$ most probable state spaces for the SCADA system. This is described in detail in [17].

4) Find the reliability bounds from equation 6 and 7.

Let

$\Xi=\left\{\begin{array}{cc}1 & \text { remote control through SCADA is possible } \\ 0 & \text { is not }\end{array}\right.$

The probability that a remote operation of $C B_{i}$ will succeed through the SCADA network is then bounded by

$$
K_{L i} \leq P(\Xi=1) \leq K_{U i}
$$

\section{Reliability TO OPERATE CB}

As a summary, the probability at time $t$ after a power outage that a manual operation of $\mathrm{CB}$ will succeed is given by $P(\Theta=$ 1 ), and the probability that a remote control of $\mathrm{CB}$ through the SCADA network will succeed is given by

$P(\Xi=1)$. The probability that a $\mathrm{CB}$ can be operated at time $t$ after a power outage is therefore given by

$$
P(\Omega=1)=1-(1-P(\Theta=1))(1-P(\Xi=1))
$$

where

$$
\Omega=\left\{\begin{array}{cc}
1 & \text { control of CB is possible } \\
0 & \text { is not }
\end{array}\right.
$$

Since $P(\Xi=1)$ is given with boundaries given by $K_{L i}$ and $K_{U i}, P(\Omega=1)$ will be bounded by

$$
\begin{aligned}
& 1-(1-P(\Theta=1))\left(1-K_{L i}\right) \leq P(\Omega=1) \leq \\
& 1-(1-P(\Theta=1))\left(1-K_{U i}\right)
\end{aligned}
$$

which is re-written as

$$
C_{L i} \leq E(X) \leq C_{U i}
$$

\section{Case Study}

To examine the ability to operate CB the distributed SCADA architectures presented in [4] for a IEEE reliability test system is used in the study, see figure 5. The SCADA system contains two control centers, National Control Center (NCC) and Area Control Center (ACC). It is assumed that the substations runs on backup power from lead-acid batteries presented in section two. It is also assumed that a remote control of CB can be performed manually in case of a failure in the remote control. For a manual operation of $\mathrm{CB}$ a call with instructions has to be directed from the TSO head-quarter to field personnel through the GSM network. Table 1 describes the components and their associated availability used in the SCADA network. Table 2 shows the expected value and the variance for the battery capacities used in the study.

TABLE I

SCADA RELIABILITY MODEL WITH COMPONENT FUNCTION DEFINITIONS AND DESIGN LEVEL AVAILABILITY.

\begin{tabular}{lll}
\hline Item & Description & Availability \\
\hline MS & Manual control handover process & 0.95 \\
WS & $\begin{array}{l}\text { Operator workstation, associated software } \\
\text { and maintenance procedures }\end{array}$ & 0.995 \\
& \\
EN & Ethernet & 0.9999 \\
BE & Backend computer system, associated & 0.95 \\
& software and maintenance procedures & \\
FE & Front end computer, software and communication & 0.99999 \\
& interfaces to RTU communications & 0.9999 \\
DM & Data circuit multiplexer & 0.9999 \\
RTU & SCADA remote terminal unit & 1.0 \\
C & Logical connection between components & 0.999 \\
DMR & Digital microwave radio link & 0.995 \\
PLC & Power line carrier & 1.0 \\
V & V25 (or RS232) direct physical connection & $P(\Phi=1)$ \\
BP & Batteries & \\
\hline
\end{tabular}




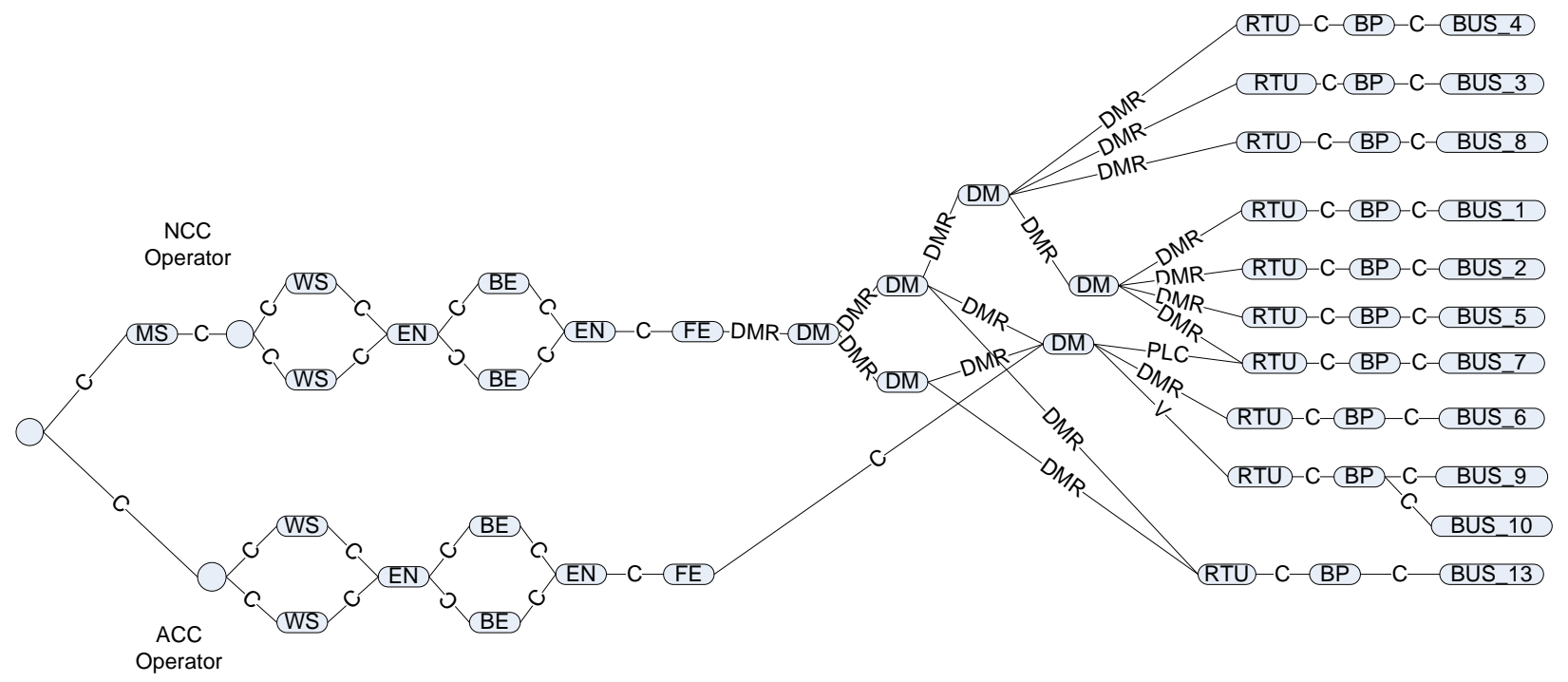

Fig. 3. Reliability model for a SCADA system

TABLE II

THE EXPECTED VALUE AND THE VARIANCE FOR THE BATTERY CAPACITIES

\begin{tabular}{lll}
\hline Battery & $E_{B}(X)$ & $V_{B}(X)$ \\
\hline Substation & 5 & 2 \\
BTS & 2 & 0.2 \\
\hline
\end{tabular}

Here it is expected that the batteries at the substations can support the load for five hours and that the batteries at BTS's can support the load for two hours. Bus one, two, three, four, five and eight can only be operated from the NCC while bus six, seven, nine, teen and thirteen can be operated from both the NCC and the ACC. To examine the difference in reliability bus four and bus seven are chosen in the study.

\section{A. Results}

Figure 6 shows the reliability to operate CB at bus four.

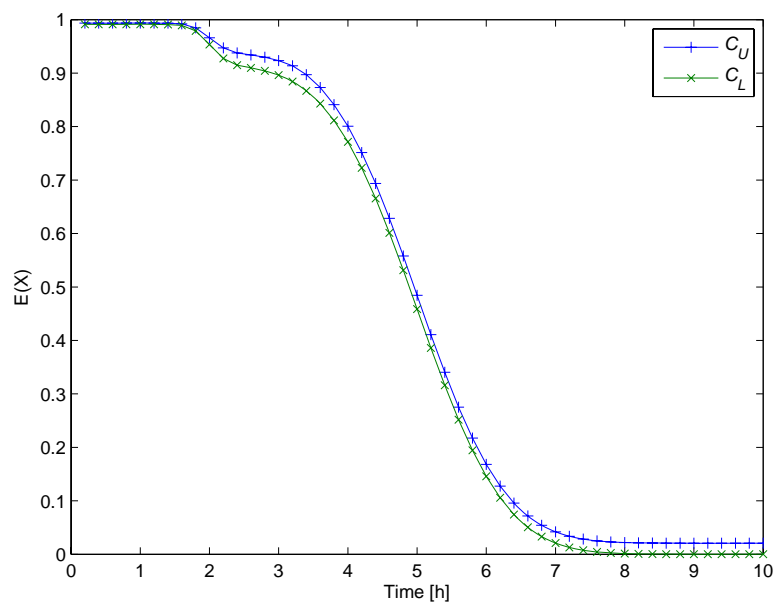

Fig. 4. Reliability to operate the $\mathrm{CB}$ at bus four
Figure 7 shows the reliability to operate $\mathrm{CB}$ at bus seven.

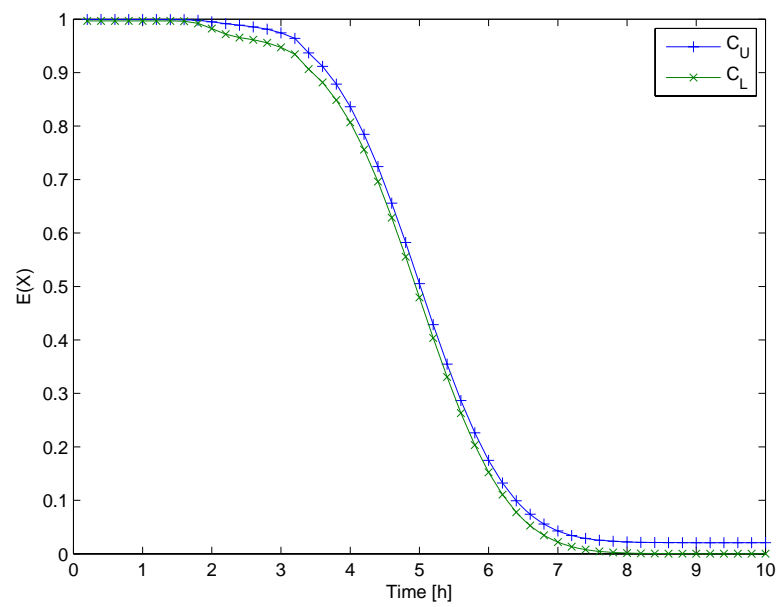

Fig. 5. Reliability to operate the $\mathrm{CB}$ at bus seven

The reliability to operate $\mathrm{CB}$ at bus four and seven is relative high for both cases the first two hours. However, when the batteries at the BTS no longer can support the load the reliability to operate $\mathrm{CB}$ at bus four decreases significant. To increase the probability of succeeding with a restoration it is therefore better in this case to operate bus four before bus seven due to architecture in the SCADA system.

\section{CONCLUSION}

A method to quantify the reliability to operate CB considering risk of communication outage during a black out has been presented. In a numerical example a SCADA architecture were applied to an IEEE reliability test system. The case study showed the impact on reliability in operating CB for both the architecture of the SCADA system and possibility to operate CB manually. In the case study the GSM network was only expected to work for two hours. The impact of this is that 
a restoration might become more time consuming when the batteries at the substation can no longer support the load, since the telecommunication network is also down. In a restoration situation it might therefore be important to operate buses with low reliability while there exists a communication channel to field personnel. The model can also be used to determine how fast a restoration must proceed to avoid issues associated with limited backup power.

For a further study a more profound reliability model of the GSM network and the PSTN should be implemented. This model could include the increase of traffic that has to be routed during a black out. It is also common to have diesel generators at large substations and in telecommunication network as an extra backup to batteries. During extended black outs the diesel distribution to the generators might become important.

\section{REFERENCES}

[1] J. McCready. "Ice Storm 1998: Lessons learned". Eastern Ontario Model Forest, 2004.

[2] M. M. Adibi, editor. Power System Restoration. IEEE Press, 2000.

[3] F. Edström and L. Söder. "Impact of Remote Control Failure on Power System Restoration Time". IEEE Conference on Probabilistic Methods Applied to Power System, Pages: 343 - 347 , 2010.

[4] A.G. Bruce. "Reliability Analysis of Electric Utility Scada System". IEEE Transactions on power systems, Volume: 13 , Issue: 3 Pages: 844 - $849,1998$.

[5] M.M. Adibi, L.H. Fink, C.J. Andrews, F. Arsanjani, M.W. Lanier, J.M. Miller, T.A. Volkmann and J. Wrubel. "Special Considerations in Power System Restoration". IEEE Transanaction on Power Systems, Volume: 12 , Issue: $11,1992$.

[6] M.M. Adibi, P. Clelland, L. Fink, H. Happ, R. Kafka, J. Raine, D. Scheurer and F. Trefny. "Power System Restoration - A Task Force Report". IEEE Transanaction on Power Systems, Volume: 2 , Issue: 2 , Pages: $271-277,1987$.

[7] M.M. Adibi, J.N. Borkoski and R.J. Kafka. "Power System Restoration The Second Task Force Report". IEEE Transanaction on Power Systems, Volume: 2 , Issue: 4 , Pages: 927 - $932,1987$.

[8] R.J. Kafka, D.R. Penders, S.H. Bouchey and M.M. Adibi. "System Restoration Plan Development for a Metropolitan Electric System". IEEE Transaction on Power Apparatus and Systems, Volume: PER-1, Issue: 8 , Pages: 33 - $33,1981$.

[9] M.M. Adibi and L.H. Fink. "Power System Restoration Planning". IEEE Transaction on Power Systems, Volume: 9 , Issue: 1 , Pages: 22 - 28, 1994.

[10] J. Nelson and W. Bolin. "Basics and Advances in Battery Systems". IEEE Transactions on industry applications, Volume: 31, Issue: 2 , Pages: 419 - $428,1995$.

[11] W.T. Grant. "Integrated Power Supplies for International Telecommunication". Electronics and Power, Volume: 26 , Issue: 8 , Pages: 652 $655,1980$.

[12] G. Rami, T. Tran-Quoc, N. Hadjsaid and J.L. Mertz. "Energy Supply for Remote Base Transceiver Stations of Telecommunication". Power Engineering Society General Meeting, Volume: 2, Pages: 1916 - 1921 , 2004.

[13] IEEE Recommended Practice for Sizing Large Led Storage Batteries for Generating Stations and Substations IEEE Std 485-1983.

[14] M. Rahnema. "Overview of the GSM System and Protol Architecture". IEEE Communications Magazine, Volume: 31 , Issue: 4 , Pages: 92 $100,1993$.

[15] C. Peersman, S. Cvetkovic, P. Griffiths and H. Spear. "The Global System for Mobile Communications Short Message Service". IEEE Personal Communications, Volume: 7 , Issue: 3 , Pages: 15 - 23, 2000.

[16] T. Ogata and J. Yamaki. "Battery Research for Telecommunications Power Supply Systems at NTT". Telecommunications Energy Conference, Pages: 697 - 703 , 1995.

[17] Y. Lam and V. Li. "An Improved Algorithm for Performance Analysis of Networks with Unreliable Components". IEEE Transactions on Communications, Volume: 34 , Issue: 5 , Pages: 496 - 497 , 1986. 